Adjustments for age, gender, systolic blood pressure, fasting plasma glucose, $\gamma$-glutamyl transpeptidase, high-density lipoprotein, triglycerides, total cholesterol and albumin, nonalcoholic fatty liver disease and prevalent atrial fibrillation remained statistically significant (OR 2.76, 95\% CI 1.32-5.77).

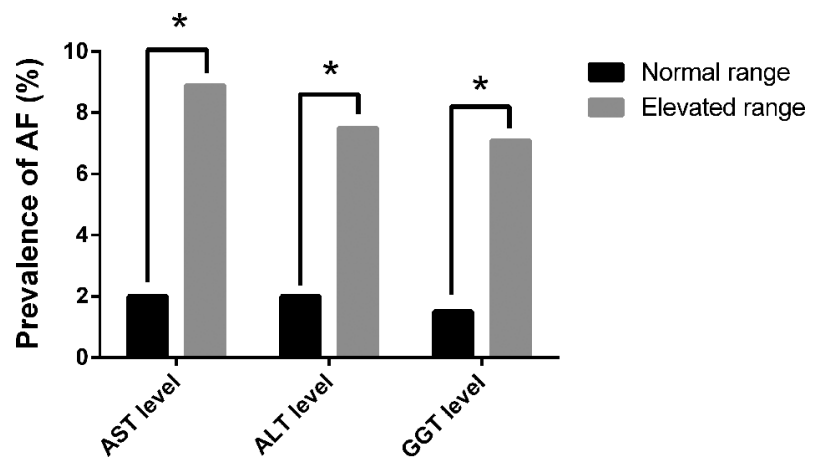

Abstract IDDF2018-ABS-0083 Figure 1

Conclusions Our results show that nonalcoholic fatty liver disease is associated with an increased risk of atrial fibrillation in an elderly Chinese population.

\section{IDDF2018-ABS-0098 PREOPERATIVE PREDICTION OF MICROVASCULAR INVASION IN HEPATOCELLULAR CANCER: A RADIOMICS MODEL USING GD-EOB-DTPA ENHANCED MRI}

${ }^{1}$ Mimi Tang*, ${ }^{2}$ Shi-Ting Feng, ${ }^{1}$ Sui Peng, ${ }^{3}$ Ming Kuang. 'Department of Gastroenterology, The First Affiliated Hospital of Sun Yat-sen University, Guangzhou, China; ${ }^{2}$ Department of Radiology, The First Affiliated Hospital of Sun Yat-sen University, China; ${ }^{3}$ Department of Liver Surgery, The First Affiliated Hospital of Sun Yat-sen University, China

\subsection{6/gutjnl-2018-IDDFabstracts.206}

Background Preoperative prediction of microvascular invasion (MVI) in patients with hepatocellular cancer (HCC) is important for surgery strategy making. We aimed to develop and validate a combined intratumoral, and peritumoral radiomics model based on Gadolinium-ethoxybenzyl-diethylenetriamine (Gd-EOB-DTPA) enhanced magnetic resonance imaging (MRI) for preoperative prediction of MVI in primary HCC patients. Methods This study included a training cohort of 110 HCC patients and a validating cohort of 50 HCC patients. All the patients underwent preoperative Gd-EOB-DTPA enhanced MRI examination and curative hepatectomy. The volumes of interest (VOIs) around the hepatic lesions including intratumoral and peritumoral regions were manually delineated in the hepatobiliary phase of MRI images, from which quantitative features were extracted and analysed. In the training cohort, the machine-learning method was applied to dimensionality reduction and selection of the extracted features.

Results The proportion of MVI-positive patients was 38.2\% and $40.0 \%$ in the training and validation cohort respectively. Supervised machine-learning selected ten features to establish a predictive model for MVI. The area under the receiver operating characteristic curve (AUC), sensitivity, specificity of the combined intratumoral and peritumoral radiomics model in the training and validation cohort were 0.85 (95\% confidence interval $[C I], 0.77-0.93), 88.2 \%, 76.2 \%$ and $0.83(95 \%$ CI, 0.71-0.95), 90.0\%, 75.0\%, respectively.

Conclusions We evaluate quantitative Gd-EOB-DTPA enhanced MRI image features of both intratumoral and peritumoral regions and provided an effective radiomics-based model for prediction of MVI in HCC patients, and may, therefore, assist clinicians to make precise treatment decision before the surgery.

\section{IDDF2018-ABS-0105 NO RESISTANCE TO TENOFOVIR ALAFENAMIDE DETECTED THROUGH 96 WEEKS OF TREATMENT IN PATIENTS WITH CHRONIC HEPATITIS B}

${ }^{1}$ Henry Lik-Yeun Chan*, ${ }^{2}$ Patrick Marcellin, ${ }^{3}$ Andrew L Cathcart, ${ }^{3}$ Neeru Bhardwaj, ${ }^{3}$ John F Flaherty, ${ }^{3}$ Anuj Gaggar, ${ }^{4}$ Namiki Izumi, ${ }^{5}$ Young-Suk Lim. ${ }^{1}$ Department of Medicine and Therapeutics, Institute of Digestive Disease and State Key Laboratory of Digestive Disease, The Chinese University of Hong Kong, Hong Kong; ${ }^{2}$ Service d'Hepatologie, Hopital Beaujon, Clichy, France; ${ }^{3}$ Gilead Sciences ,Foster City, CA, USA; ${ }^{4}$ Department of Gastroenterology and Hepatology, Musashino Red Cross Hospital, Tokyo, Japan; ${ }^{5}$ Department of Gastroenterology, Asan Medical Center, Seoul, Korea, South

\subsection{6/gutjnl-2018-IDDFabstracts.207}

Background Presented herein are the post Week 48 through Week 96 resistance analyses for 2 Phase 3 studies evaluating tenofovir alafenamide (TAF) versus tenofovir disoproxil fumarate(TDF) for the treatment of chronic hepatitis $\mathrm{B}(\mathrm{CHB})$ in HBeAg + and HBeAg- adults.

Methods HBV pol/RT population or deep sequencing was conducted for patients with $\geq 24$ weeks of treatment with viremia (HBV DNA $\geq 69 \mathrm{IU} / \mathrm{mL}$ ) at Week 96 or at early discontinuation post Week 48. Deep sequencing was conducted for patients with HBV DNA $>159 \mathrm{IU} / \mathrm{mL}$ and sequence changes at the consensus sequence level (15\%) are reported. Virologic breakthrough (VB) was defined as $\mathrm{HBV}$ DNA $\geq 69 \mathrm{IU} / \mathrm{mL}$ after achieving $<69 \mathrm{IU} / \mathrm{mL}$ or $\mathrm{a} \geq 1.0-\log _{10}$ increase from nadir. Phenotypic analysis using recombinant HBV in HepG2 cells was performed for VB patients who were adherent to study drug (plasma drug levels), patients with conserved site substitutions, or for polymorphic substitutions emergent in $>1$ patient.

Results 1298 patients were randomised (TAF: $n=866$; TDF: $\mathrm{n}=432$ ). A similar percentage of patients in the TAF or TDF arms qualified for sequence analysis post Week 48 through Week 96 of treatment (TAF: 10.5\%, TDF: 10.9\%). In the TAF arm, 87 patients qualified at Week 96: 32 had polymorphic site substitutions, and 9 had conserved site substitutions. In the TDF arm, 45 patients qualified at Week $9611 \mathrm{had}$ polymorphic site substitutions, and 2 had conserved site substitutions. At Week 96, a small percentage of patients experienced VB (TAF: 2.4\%, TDF: 3.0\%), and VB was often associated with documented study drug nonadherence (TAF: 22\%, TDF: 46\%). 27 patients qualified for phenotypic analysis post Week 48 through Week 96 (TAF: $n=19$, TDF: $n=8$ ) and no patient isolates tested showed a reduction in susceptibility to TAF or tenofovir, respectively. 